\title{
PLANETARY SYSTEMS AROUND CLOSE BINARY STARS: THE CASE OF THE VERY DUSTY, SUN-LIKE, SPECTROSCOPIC BINARY BD+20 307
}

\author{
B. Zuckerman, ${ }^{1,2}$ Francis C. Ferel, ${ }^{3}$ Michael H. Williamson, ${ }^{3}$ Gregory W. Henry, ${ }^{3}$ and M. P. Muno ${ }^{4}$ \\ Received 2008 January 12; accepted 2008 August 8
}

\begin{abstract}
Field star BD+20 307 is the dustiest known main-sequence star, based on the fraction of its bolometric luminosity, $\sim 4 \%$, that is emitted at infrared wavelengths. The particles that carry this large infrared luminosity are unusually warm, comparable to the temperature of the zodiacal dust in the solar system, and their existence is likely to be a consequence of a fairly recent collision of large objects such as planets or planetary embryos. Thus, the age of BD+20 307 is potentially of interest in constraining the era of terrestrial planet formation. The present project was initiated with an attempt to derive this age using the Chandra X-Ray Observatory to measure the X-ray flux of BD+20 307 in conjunction with extensive photometric and spectroscopic monitoring observations from Fairborn Observatory. However, the recent realization that $\mathrm{BD}+20307$ is a short-period, double-line, spectroscopic binary whose components have very different lithium abundances vitiates standard methods of age determination. We find the system to be metalpoor; this, combined with its measured lithium abundances, indicates that $\mathrm{BD}+20307$ may be several to many Gyr old. BD+20 307 affords astronomy a rare peek into a mature planetary system in orbit around a close binary star (because such systems are not amenable to study by the precision radial velocity technique).
\end{abstract}

Subject headings: planetary systems: formation — stars: individual (BD+20 307) — stars: rotation X-rays: stars

Online material: machine-readable table

\section{INTRODUCTION}

There are hundreds of main-sequence stars that are known to have orbiting dusty debris disks. The dust in most of these systems is cold and appears to be analogous to dust in the Sun's Kuiper Belt. Only a small percentage of debris disks contain warm dust analogous to that in the Sun's asteroid belt and zodiacal cloud (e.g., Su et al. 2006; Rhee et al. 2008; Trilling et al. 2008; Smith et al. 2008). For stars that are at least 100 Myr old, the warm dust luminosity $\left(L_{\mathrm{IR}} / L_{\mathrm{bol}}\right)$ is typically a few times $10^{-4}$ or less. Smith et al. (2008) discuss and review the significance of this warm, mid-infrared, emission in the context of the Sun's zodiacal dust cloud. They note that Spitzer Infrared Observatory photometry (Hines et al. 2006; Bryden et al. 2006) indicates that warm dust is detected at only $2 \% \pm 2 \%$ of the observed systems. A more recent, more comprehensive, Spitzer survey (Trilling et al. 2008) yielded a similar small percentage of warm excess emission stars in a sample of $213 \mathrm{~F}$ - and G-type stars. A comparable small percentage also applies to warm excess A-type stars of age $>400 \mathrm{Myr}$ (Su et al. 2006), which, as will be seen below, is the age range of interest in the present paper. In all of these surveys and in other published Spitzer surveys, the typical midinfrared luminosity is sufficiently small that the warm dust could be produced by collisions of asteroids; for example, in a massive asteroid belt perturbed by the gravitational field of a nearby giant planet.

In contrast, Rhee et al. (2008) discuss the dustiest mainsequence stars currently known: Pleiad HD 23514 and, dustiest

\footnotetext{
${ }^{1}$ Department of Physics and Astronomy, University of California, Los Angeles, CA 90095.

2 UCLA Center for Astrobiology.

3 Center of Excellence in Information Systems, Tennessee State University, 3500 John A. Merritt Boulevard, Box 9501, Nashville, TN 37209.

4 Space Radiation Laboratory, California Institute of Technology, Pasadena, CA $91125-4700$.
}

of all, the G0 field dwarf BD+20 307 (HIP 8920; Song et al. 2005; Weinberger 2008; Weinberger et al. 2008). The dust luminosity at these two stars is orders of magnitude larger than at other mid-infrared excess main-sequence stars, and collisions of asteroids seem quite inadequate to explain the data. A plausible model to account for so much dust so close to these stars involves a fairly recent collision of two planets or planetary embryos in the terrestrial planet zone (Rhee et al. 2008).

$\mathrm{BD}+20307$ was originally presumed to be a single star, and a measurement of its (presumably youthful) age would have constrained the duration of the era during which major collisional events occur in youthful planetary systems. Using photospheric lithium content, Galactic space motion, and an upper limit to the X-ray flux measured with the ROSAT All-Sky Survey, Song et al. (2005) estimated the age of BD+20 307 to be 300 Myr. To refine this estimate, we obtained X-ray measurements with the Chandra X-Ray Observatory and hundreds of photometric optical observations to measure the star's rotation period and age, employing the technique of gyrochronology (Barnes 2007). While we were carrying out these observations, Weinberger (2008) obtained the surprising result that $\mathrm{BD}+20307$ is a short-period ( $\sim 3.5$ day) spectroscopic binary of two nearly identical stars. The reason this was a surprise is that the Song et al. (2005) echelle spectrum showed no evidence of two stars; by a low-probability $(<10 \%)$ quirk of fate, their 2004 epoch spectrum was obtained at a time when the two stars had nearly the same radial velocities.

While Spitzer surveys for debris disks around stars have often explicitly omitted binaries, Trilling et al. (2007) found small quantities of cool dust around a substantial fraction of binary stars. However, by analogy with the discussion of single stars in the first paragraph above, dust around such binary stars can readily be accounted for by collisions of Kuiper Belt objects.

On learning of the Weinberger (2008) result, we initiated a program to monitor the radial velocity of $\mathrm{BD}+20307$. Our various observations are described in the next section. This is followed 
TABLE 1

Individual Photometric Observations of BD+20 307

\begin{tabular}{|c|c|c|c|c|c|c|}
\hline $\begin{array}{c}\text { Hel. Julian Date } \\
\text { (HJD }-2,400,000)\end{array}$ & $\begin{array}{l}D-A \\
(\mathrm{mag})\end{array}$ & $\begin{array}{r}D-B \\
(\mathrm{mag})\end{array}$ & $\begin{array}{l}D-C \\
\text { (mag) }\end{array}$ & $\begin{array}{r}C-A \\
(\mathrm{mag})\end{array}$ & $\begin{array}{l}C-B \\
(\mathrm{mag})\end{array}$ & $\begin{array}{r}B-A \\
(\mathrm{mag})\end{array}$ \\
\hline $53,402.5866 \ldots \ldots \ldots \ldots \ldots \ldots \ldots$ & 2.4388 & 2.0525 & 1.1812 & 1.2576 & 0.8712 & 0.3864 \\
\hline $53,402.5922 \ldots \ldots \ldots \ldots \ldots \ldots \ldots$ & 2.4401 & 2.0518 & 1.1816 & 1.2585 & 0.8703 & 0.3881 \\
\hline $53,404.5932 \ldots \ldots \ldots \ldots \ldots \ldots \ldots$ & 2.4366 & 2.0495 & 1.1767 & 1.2598 & 0.8728 & 0.3871 \\
\hline $53,405.5882 \ldots \ldots \ldots \ldots \ldots \ldots \ldots$ & 2.4385 & 2.0507 & 1.1799 & 1.2586 & 0.8708 & 0.3878 \\
\hline $53,405.5939$ & 2.4363 & 2.0516 & 1.1766 & 1.2598 & 0.8750 & 0.3848 \\
\hline
\end{tabular}

NoTEs.-Differential magnitudes were measured in the Strömgren $(b+y) / 2$ passband. Table 1 is published in its entirety in the electronic edition of the Astrophysical Journal. A portion is shown here for guidance regarding its form and content.

by a discussion of the age and some other aspects of this exceptionally dusty binary star, which points to the existence of rocky planets in the terrestrial planet region.

\section{OBSERVATIONS}

\subsection{X-Rays}

We observed BD+20 307 with the Chandra X-Ray Observatory Advanced CCD Spectrometer Spectroscopic array (ACIS-S; Weisskopf et al. 2002) starting on 2007 September 25 at 23:34:20 (UT), for a total of $4919 \mathrm{~s}$. We reduced the event lists using the Chandra Interactive Analysis of Observations (CIAO) software version 3.4 and the calibration database version 3.4.1. We filtered the data using the standard techniques described on the Web site of the Chandra X-Ray Center (CXC).

We then made images of the events in the full bandpass of $0.5-8.0 \mathrm{keV}$, a soft $0.5-2.0 \mathrm{keV}$ band to provide sensitivity to foreground sources, and a hard $4-8 \mathrm{keV}$ band to provide sensitivity to highly absorbed sources. We searched these for point sources using the wavelet-based algorithm wavdetect (Freeman et al. 2002). Images were made for two spatial scales: one $1024 \times$ 1024 pixel image covering the central portion of the field at the $0.5^{\prime \prime}$ resolution of the detector, and one image at $2^{\prime \prime}$ resolution that covered all of the active detectors. We identified a total of 30 sources. The majority of these, 26 , were detected strongly in the $0.5-2 \mathrm{keV}$ band. Only four sources were detected in the full band but not in the soft band, and no sources were detected exclusively in the hard band. Three of the brightest sources had counterparts in the $2 \mathrm{MASS}$ catalog, so we used these to register the absolute astrometry for the observation. We found that the $\mathrm{X}$-ray sources were offset by an average of $-0.24^{\prime \prime} \pm 0.17^{\prime \prime}$ in right ascension, and $+0.36^{\prime \prime} \pm 0.17^{\prime \prime}$ in declination, and applied this shift to the locations of the X-ray sources.

We found an X-ray source coincident with BD+20 307 at $01^{\mathrm{h}} 54^{\mathrm{m}} 50.37^{\mathrm{s}},+21^{\circ} 18^{\prime} 22.3^{\prime \prime}$, with an uncertainty of $0.4^{\prime \prime}$ according to Equation (5) of Hong et al. (2005). We extracted photometry and light curves for the source using the acis_extract routine. ${ }^{5}$ Events for the source were extracted from a circle with radius of 2.5 pixels, which corresponds to $90 \%$ of the pointspread function at $1.4 \mathrm{keV}$. Background was derived from an annular 25 pixel region, excluding a circle of 5 pixels around the point source. We found $158 \pm 12$ net counts from BD+20 307, all of which arrived in the $0.5-2.0 \mathrm{keV}$ band. In total, only four sources in the $340 \operatorname{arcmin}^{2}$ field of view had $>100$ net counts, so we conclude that this X-ray source is BD+20 307.

Next, we binned the events over energy to obtain spectra of the source and background, and obtained the detector response function and effective area using standard CIAO tools. We mod- eled the spectrum as a thermal plasma ${ }^{6}$ using XSPEC version 12.2.1 (Arnaud 1996). We used the Cash statistic (Cash 1979) to identify a best fit and $1 \sigma$ uncertainty on the model parameters, and found a temperature $k T=0.61_{-0.02}^{+0.06} \mathrm{keV}$ and a flux of $1.1 \times$ $10^{-13} \mathrm{erg} \mathrm{cm}^{-2} \mathrm{~s}^{-1}(0.5-2.0 \mathrm{keV})$. Finally, we tested for variability by comparing the arrival times of the photons to a uniform distribution using the Kolmogorov-Smirnov statistic, and found that the data were consistent with a constant flux at the $76 \%$ confidence level.

\subsection{Optical Photometry}

We used the T12 $0.8 \mathrm{~m}$ Automatic Photometric Telescope (APT) located at Fairborn Observatory in Arizona to collect a total of 449 nightly brightness measurements of BD+20 307 between 2005 February and 2007 December. Such measurements of solar-type stars often reveal brightness variations caused by cool, dark photospheric spots as they are carried into and out of view by the star's rotation (e.g., Radick et al. 1987). Our APTs have proven to be very successful at measuring stellar rotation periods, even for stars with low photometric amplitudes of a few hundredths of a magnitude or less (e.g., see Henry et al. 1995).

We programmed the T12 APT to observe BD+20 307 differentially with respect to three comparison stars. Its two-channel precision photometer observed each star simultaneously in the standard Strömgren $b$ and $y$ passbands. The three comparison stars, designated A, B, and C, respectively, are HD $12354(V=$ $6.61, B-V=0.34, \mathrm{~F} 1 \mathrm{IV}-\mathrm{V})$, HD $12846(V=6.89, B-V=$ $0.66, \mathrm{G} 2 \mathrm{~V})$, and HD $10088(V=7.89, B-V=0.31$, F0 IV $)$, while $\mathrm{BD}+20307(V=8.98, B-V=0.56$, F9 V) is designated as star $\mathrm{D}$. The observations were reduced to form the six differential magnitudes $D-A, D-B, D-C, C-A, C-B$, and $B-A$. To increase the precision of our measurements, we combined the Strömgren $b$ and $y$ differential magnitudes into a single $(b+y) / 2$ passband for each of the six differential magnitudes. For additional information on the telescope, photometer, observing procedures, data reduction techniques, and photometric precision, see Henry (1999) and Eaton et al. (2003).

The individual reduced and standardized $(b+y) / 2$ differential magnitudes of BD+20 307 are listed in Table 1, where they will be available for future studies such as long-term spot evolution or for comparison with other activity indicators in BD+20 307. To improve our measurement precision still further, we averaged the three $D-A, D-B$, and $D-C$ differential magnitudes of $\mathrm{BD}+20307$ into a single value representing the difference in brightness between $\mathrm{BD}+20307$ and the mean of the three comparison stars $[D-(A+B+C) / 3]$, which we refer to as the ensemble average. The standard deviations of the three comparison 

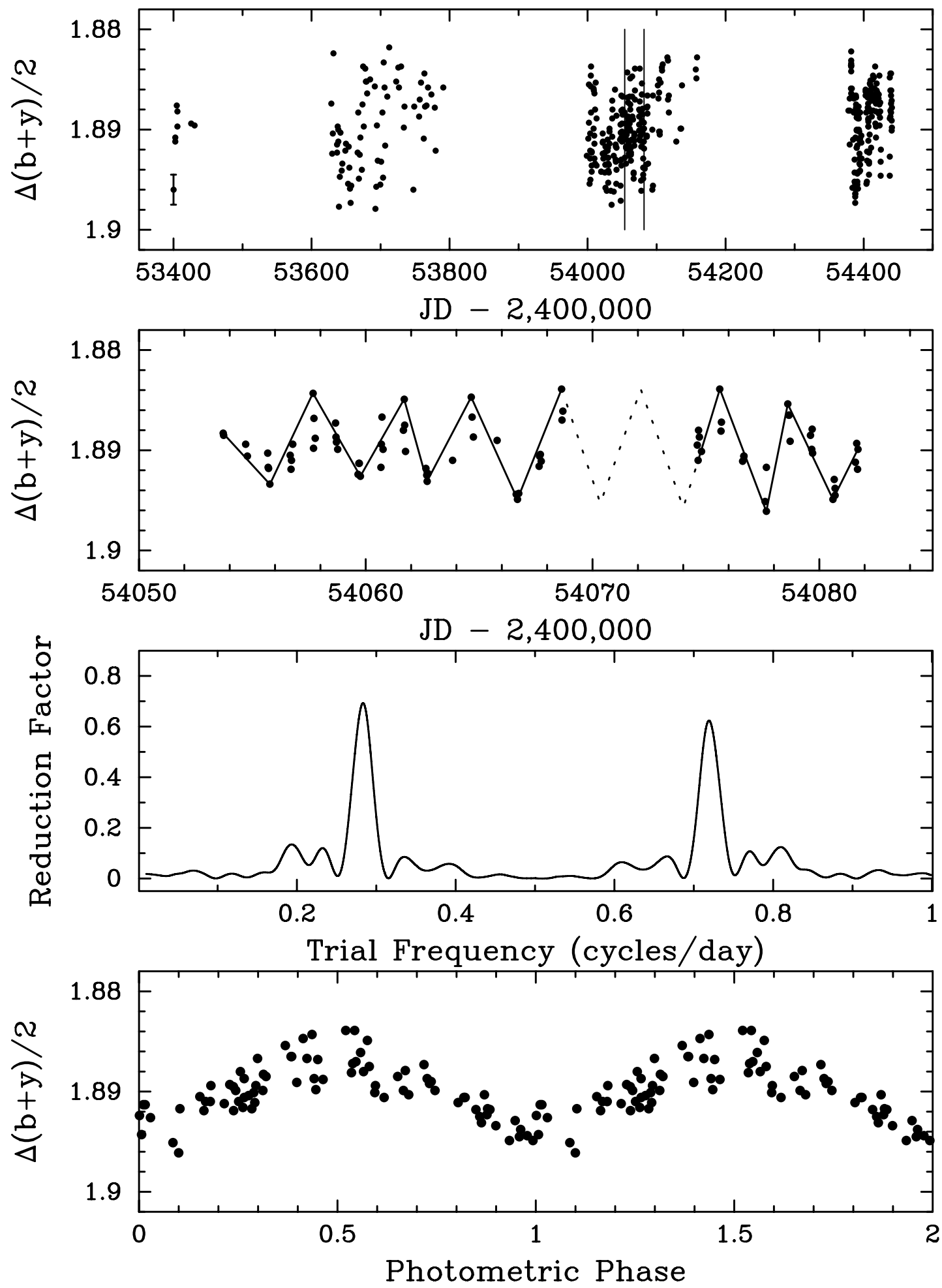

FIG. 1.-Top: Ensemble photometric observations of BD +20307 taken in the Strömgren $(b+y) / 2$ passband with the T12 $0.8 \mathrm{~m}$ APT at Fairborn Observatory over four observing seasons. The error bar in the lower left corner represents the typical $1 \sigma$ uncertainty $( \pm 0.0015 \mathrm{mag})$ for a single observation. Second panel: Portion of the third observing season, marked with the vertical lines in the top panel and plotted with an expanded abscissa, shows low-amplitude but coherent variability in BD+20 307. Third panel: Power spectrum of the data in the second panel revealing a best period of 3.530 days. Bottom: Plot of the data in the second panel phased with the 3.530 day period and a computed time of minimum. The amplitude of brightness variation is only $0.0066 \mathrm{mag}$.

star differentials $C-A, C-B$, and $B-A$ from Table 1 all lie between 0.001 and $0.002 \mathrm{mag}$, which is typical of the measurement precision with the T12 APT. Therefore, all three comparison stars are constant from night to night to the limit of our precision.

The 449 ensemble differential magnitudes of BD+20 307 in our $(b+y) / 2$ passband are plotted in the top panel of Figure 1, where they fall into four separate observing seasons. No significant periodicity was found when analyzing the observing seasons separately or the data set as a whole. Therefore, any photospheric starspots on either component of BD+20 307 must have lifetimes of only a few weeks at most. Indeed, a small portion of the third observing season, marked by the pair of vertical lines in the top 
panel of Figure 1 and shown with an expanded abscissa in the second panel, does appear to reveal a few cycles of low-amplitude but coherent brightness variability. The brightest and faintest observations of each cycle are connected with straight line segments to guide the eye; the interpolation of approximately one and a half missing cycles is shown by the dotted line segments.

The third panel of Figure 1 shows the power spectrum of the data from the second panel, computed with the method of Vaníček (1971). This period-finding technique uses least squares to fit the data with sine curves over a range of trial periods and measures the resulting reduction in the variance of the data for each trial frequency. The strongest peak occurs at a frequency of $0.2833 \pm$ 0.0020 day $^{-1}$, corresponding to a period of $3.530 \pm 0.025$ days. The lower peak is a one-day alias of this period. Analysis of small sections of the fourth season light curve provides support for the 3.53 day period, yielding less precise period values between 3.28 and 3.44 days.

The bottom panel of Figure 1 shows the data from the second panel phased with the 3.53 day photometric period and a time of brightness minimum of JD 2,454,003.18 \pm 0.04 days, computed from a least-squares sine fit to the phase curve. The sine fit gives a peak-to-peak amplitude of $0.0066 \pm 0.0005 \mathrm{mag}$ and an $\mathrm{rms}$ to the fit of 0.0014 mag, which approximates the typical precision of our photometric measurements.

\subsection{Optical Spectroscopy}

From 2008 January through March we obtained 28 spectrograms of BD+20 307 with the Tennessee State University $2 \mathrm{~m}$ automatic spectroscopic telescope (AST), fiber-fed echelle spectrograph, and a $2048 \times 4096$ SITe ST-002A CCD. The echelle spectrograms have 21 orders, covering the wavelength range 4920-7100 $\AA$ with an average resolution of $0.17 \AA$. The typical signal-to-noise ratio of these observations is $\sim 10$. Eaton $\&$ Williamson $(2004,2007)$ have given a more extensive description of the telescope and spectrograph, which are operated at Fairborn Observatory.

For the AST spectra, lines in approximately 170 regions, centered on the rest wavelengths (Moore et al. 1966) of relatively strong, mostly $\mathrm{Fe}$ I lines that were not extensively blended with other nearby strong features were measured. Lines at the ends of each echelle order were excluded because of their lower signalto-noise ratios. The wavelength scale of each spectrum was initially determined from Th-Ar comparison spectra obtained at the beginning and end of the night, and that scale was refined with the use of the telluric $\mathrm{O}_{2}$ lines near $6900 \AA$, which are in each stellar spectrum. A Gaussian function was fitted to the profile of each component. If lines of the two components were blended, a double Gaussian was used to fit the combined profile. The difference between the observed wavelength and that given in the solar line list of Moore et al. (1966) was used to compute the radial velocity, and a heliocentric correction was applied. The final mean velocity for each component is given in Table 2. Our unpublished velocities of several IAU standard solar-type stars indicate that the Fairborn Observatory velocities have a small zero-point offset of $-0.3 \mathrm{~km} \mathrm{~s}^{-1}$ relative to the velocities of Scarfe et al. (1990).

There is no confusion concerning component identification because the line strengths are different enough, as discussed in the next paragraph, so that the primary and secondary can be identified visually. With our photometric period adopted and $0.3 \mathrm{~km} \mathrm{~s}^{-1}$ added to each Fairborn Observatory velocity, initial orbital elements for the primary were computed with BISP (Wolfe et al. 1967), a program that implements a slightly modified version of the Wilsing-Russell method. The orbit was then refined with
TABLE 2

Radial Velocities of BD+20 307

\begin{tabular}{|c|c|c|c|c|c|}
\hline $\begin{array}{c}\text { Hel. Julian Date } \\
(\text { HJD }-2,400,000)\end{array}$ & Phase & $\begin{array}{c}V_{A} \\
\left(\mathrm{~km} \mathrm{~s}^{-1}\right)\end{array}$ & $\begin{array}{l}(O-C)_{A} \\
\left(\mathrm{~km} \mathrm{~s}^{-1}\right)\end{array}$ & $\begin{array}{c}V_{B} \\
\left(\mathrm{~km} \mathrm{~s}^{-1}\right)\end{array}$ & $\begin{array}{l}(O-C)_{B} \\
\left(\mathrm{~km} \mathrm{~s}^{-1}\right)\end{array}$ \\
\hline $54,495.585 \ldots$ & 0.842 & 10.8 & -0.4 & -37.1 & 0.7 \\
\hline $54,497.586 \ldots \ldots \ldots \ldots \ldots \ldots$ & 0.427 & -51.4 & -0.2 & 29.8 & 0.6 \\
\hline $54,498.586^{\mathrm{a}} \ldots \ldots \ldots \ldots \ldots \ldots$ & 0.720 & -23.4 & -2.8 & -4.8 & -1.2 \\
\hline $54,498.690^{\mathrm{a}} \ldots \ldots \ldots \ldots \ldots$ & 0.750 & -12.9 & -0.5 & -12.9 & -0.5 \\
\hline $54,499.586 \ldots \ldots \ldots \ldots \ldots \ldots . .$. & 0.012 & 30.6 & -0.1 & -58.7 & 0.0 \\
\hline $54,499.690 \ldots \ldots \ldots \ldots \ldots \ldots$ & 0.042 & 28.4 & -0.9 & -57.2 & 0.0 \\
\hline $54,502.690 \ldots$ & 0.919 & 26.1 & 0.7 & -52.6 & 0.4 \\
\hline $54,505.630^{\mathrm{a}} .$. & 0.779 & -3.4 & 1.2 & -20.0 & 0.9 \\
\hline $54,505.679^{\mathrm{a}} \ldots \ldots \ldots \ldots \ldots$ & 0.793 & -1.6 & -0.8 & -24.6 & 0.3 \\
\hline $54,506.630 \ldots \ldots \ldots \ldots \ldots \ldots$ & 0.071 & 26.2 & -0.3 & -54.3 & -0.1 \\
\hline $54,506.679 \ldots \ldots \ldots \ldots \ldots \ldots$ & 0.086 & 24.9 & 0.3 & -52.2 & 0.0 \\
\hline $54,507.590 \ldots \ldots \ldots \ldots \ldots \ldots$ & 0.352 & -38.2 & 0.1 & 14.7 & -0.7 \\
\hline $54,508.590 \ldots \ldots \ldots \ldots \ldots \ldots$ & 0.645 & -38.0 & 1.0 & 16.6 & 0.5 \\
\hline $54,510.591^{\mathrm{a}} \ldots \ldots \ldots \ldots \ldots \ldots$ & 0.230 & -3.3 & 3.6 & -22.2 & -3.9 \\
\hline $54,514.593 \ldots \ldots \ldots \ldots \ldots \ldots . .$. & 0.400 & -47.7 & -0.4 & 25.3 & 0.2 \\
\hline $54,516.594 \ldots \ldots \ldots \ldots \ldots \ldots$ & 0.985 & 30.1 & -0.5 & -59.4 & -0.8 \\
\hline $54,516.658 \ldots \ldots \ldots \ldots \ldots \ldots$ & 0.004 & 30.7 & -0.1 & -59.6 & -0.8 \\
\hline $54,517.594^{\mathrm{a}}$. & 0.277 & -20.4 & -0.6 & -5.7 & -1.2 \\
\hline $54,517.658^{\mathrm{a}} \ldots \ldots \ldots \ldots \ldots \ldots$ & 0.296 & -23.4 & 1.3 & 1.4 & 0.6 \\
\hline $54,522.597^{\mathrm{a}} \ldots \ldots \ldots \ldots \ldots$ & 0.740 & -12.4 & 2.7 & -12.4 & -2.9 \\
\hline $54,523.597 \ldots \ldots \ldots \ldots \ldots \ldots . . .$. & 0.032 & 29.9 & 0.0 & -58.1 & -0.2 \\
\hline $54,523.648 \ldots \ldots \ldots \ldots \ldots \ldots$ & 0.047 & 29.8 & 0.9 & -55.8 & 1.0 \\
\hline $54,524.598 \ldots \ldots \ldots \ldots \ldots \ldots$ & 0.325 & -31.6 & 0.5 & 8.8 & 0.2 \\
\hline $54,532.602 \ldots \ldots \ldots \ldots \ldots \ldots$ & 0.665 & -34.2 & 0.1 & 10.7 & -0.4 \\
\hline $54,533.604 \ldots \ldots \ldots \ldots \ldots \ldots$ & 0.958 & 29.9 & 0.6 & -56.0 & 1.2 \\
\hline $54,534.604^{\mathrm{a}}$ & 0.251 & -11.8 & 0.8 & -11.8 & 0.4 \\
\hline $54,535.604 \ldots \ldots \ldots \ldots \ldots \ldots$ & 0.543 & -54.9 & -0.8 & 31.6 & -0.7 \\
\hline $54,540.602 \ldots \ldots \ldots \ldots \ldots \ldots$ & 0.004 & 29.9 & -0.9 & -60.0 & -1.2 \\
\hline
\end{tabular}

${ }^{\text {a }}$ Spectrum has blended components, velocities given zero weight.

SB1 (Barker et al. 1967), a program that uses differential corrections. An orbit for the secondary velocities was also computed. Zero weight was assigned to the velocities of the nine observations that had blended components with velocity separations less than $30 \mathrm{~km} \mathrm{~s}^{-1}$. Based on the variances of the two solutions, weights of 1.0 and 0.9 were assigned to the primary and secondary velocities, respectively, of the 19 other spectrograms. An orbital solution of both components together was obtained with SB2, a modified version of SB1. Because the eccentricity of this solution is extremely small, $0.004 \pm 0.004$, a circular orbit was computed with SB2C (D. Barlow 1998, private communication), which also uses differential corrections to determine the elements. For this solution the weights of the velocities were the same as those previously assigned. The tests of Lucy \& Sweeney (1971) indicate that the circular orbit solution is to be preferred. The orbital phases of the observations and velocity residuals to the final computed curves are given in Table 2, and the orbital elements and related parameters are listed in Table 3. For a circular orbit the element $T$, a time of periastron passage, is undefined. So, as recommended by Batten et al. (1989), $T_{0}$, a time of maximum velocity for the primary, is given instead. In Figure 2 the observed velocities are compared with the computed velocity curves. Zero phase is a time of maximum velocity of the primary.

The components are similar in spectral type, as indicated by the best-fit effective temperatures and gravities that were determined by Weinberger (2008). Thus, for the two components the iron lines, which dominate the spectrum, and those of other elements except for lithium, which is discussed in $\S 3$, are similar in strength. To determine the magnitude difference between the two components, we followed the spectroscopic method of Petrie 
TABLE 3

Orbital Elements and Related Parameters of BD+20 307

\begin{tabular}{|c|c|}
\hline Parameter & Value \\
\hline$P$ (days) & $3.42015 \pm 0.00067$ \\
\hline$T_{0}(\mathrm{HJD})$ & $2,454,506.3855 \pm 0.0028$ \\
\hline$\gamma\left(\mathrm{km} \mathrm{s}^{-1}\right)$ & $-12.43 \pm 0.11$ \\
\hline 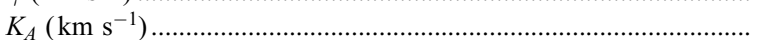 & $43.20 \pm 0.18$ \\
\hline 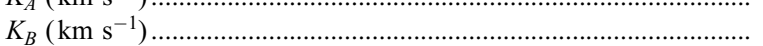 & $46.39 \pm 0.18$ \\
\hline$e_{\text {e }}$ & 0.0 (adopted) \\
\hline$M_{A} \sin ^{3} i\left(M_{\odot}\right)$ & $0.1322 \pm 0.0012$ \\
\hline$M_{B} \sin ^{3} i\left(M_{\odot}\right)$ & $0.1231 \pm 0.0011$ \\
\hline$a_{A} \sin i\left(10^{6} \mathrm{~km}\right)$ & $2.0317 \pm 0.0083$ \\
\hline$a_{B} \sin i\left(10^{6} \mathrm{~km}\right)$ & $2.1816 \pm 0.0087$ \\
\hline 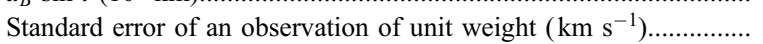 & 0.6 \\
\hline
\end{tabular}

(1939). For spectra with well-separated components we measured the ratio of the equivalent widths of over $100 \mathrm{Fe}$ I primary and secondary lines. The average of the equivalent width ratio of the secondary to primary components is $0.78 \pm 0.01$ and corresponds to the luminosity ratio of the stars. This is then converted into a very modest magnitude difference of 0.27 . Given the wavelength range of our spectra, we adopt this value as the $V$-magnitude difference. Combined with the total $V$ mag of 8.98 listed by Hipparcos, this results in $V_{A}=9.61 \mathrm{mag}$ and $V_{B}=9.88$.

Weinberger (2008) compared synthetic spectra, computed with solar abundances, to a spectrum of BD+20 307 with well-separated components and determined the effective temperatures of the two stars. The two components differed by just $250 \mathrm{~K}$, and the average of the two effective temperatures is $6375 \mathrm{~K}$, which corresponds to a $B-V$ color of 0.48 (Flower 1996). However, the observed combined $B-V$ color of the system is 0.56 (Perryman et al. 1997). This suggests either that the star has significant extinction in the visual region or the components are metal-poor. The first possibility appears to be unlikely. At a distance of only $96 \mathrm{pc}$ (van Leeuwen 2007), chances are small that sufficient interstellar matter lies between the Sun and BD+20 307 to produce a color excess of $0.08 \mathrm{mag}$ (Welsh et al. 1998; Lallement et al. 2003; Cox 2000; B. Welsh 2008, private communication). Also, little, if any, circumstellar extinction from the collision that produced the large infrared excess is expected. Collisions among the dust particles rapidly damp out the components of motion not in the orbital plane. For BD+20 307 the plane containing the collision material is presumably the same as that of the binary orbit, which has an inclination of about $31^{\circ}$ and so is much closer to face-on

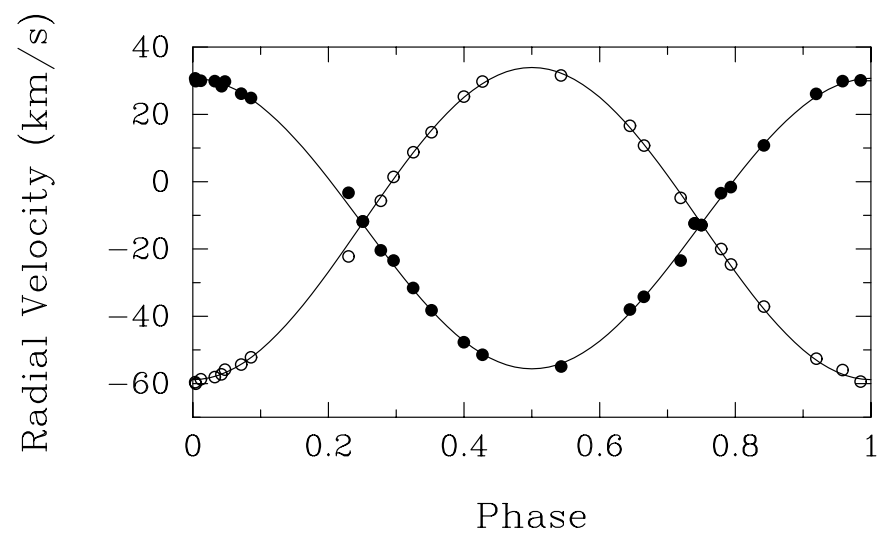

FIG. 2.-Radial velocities (dots: component A; open circles: component B) of $\mathrm{BD}+20307$ compared with the computed radial velocity curves. Zero phase is a time of maximum velocity of the primary, component $\mathrm{A}$. $\left(i=0^{\circ}\right)$ than edge-on. In addition, the spectral energy distribution of BD+20 307 is such that very little (hot) dust exists within a few tenths of an AU of the central stars (Song et al. 2005; Weinberger et al. 2008). Thus, the picture of this system is of the close binary components shining out through the central hole in the dust ring distribution.

The metal-poor alternative appears to be a more attractive explanation of the apparently discrepant $B-V$ indicated in the previous paragraph. Synthetic spectra with solar abundances require a higher effective temperature then do metal-poor stars to produce a given (weak) line strength. To investigate whether the system is metal-poor, we estimated the iron abundance for the components of $\mathrm{BD}+20307$. We compared the combined average equivalent width of components $A$ and $B$, determined from the same set of iron lines as those used for the luminosity ratio, with the average iron equivalent widths of several single dwarf stars, including $\chi \mathrm{Her}, \beta \mathrm{Com}$, and HR 7560, which have similar $B-$ $V$ values and known iron abundances. The spectra of these comparison stars were obtained with the same telescope, spectrograph, and detector as those of $\mathrm{BD}+20$ 307. The average equivalent widths of HR 7560 and $\beta \mathrm{Com}$, which have essentially solar iron abundances (Taylor 2005), are 30\% larger, but the average equivalent width of $\chi$ Her, which has $[\mathrm{Fe} / \mathrm{H}]=-0.43$ (Taylor 2003), is identical to that of $\mathrm{BD}+20307$. Thus, we conclude that $\mathrm{BD}+20307$ has a similar iron abundance and is metal-poor compared to the Sun. Given that dust particles are made of refractory metals, it does seem remarkable that the dustiest main-sequence star presently known to astronomy appears to be of low metallicity.

\section{DISCUSSION}

The initial motivation for the research reported in this paper was determination of the age of BD+20 307 and the implications of its very dusty circumstellar disk for the formation of terrestrial planets around Sun-like stars. As such, we initially obtained the X-ray and optical photometry data described in $\S \S 2.1$ and 2.2. However, the discovery that BD+20 307 is a close binary star (SB2; Weinberger 2008) greatly altered our interpretation of these data and motivated the spectroscopic observations discussed in $\S 2.3$. In the present section we consider some properties of $\mathrm{BD}+20307$ derived from the data and their relationship to its age.

Assuming BD+20 307 is a single star, from a fit to its spectral energy distribution, Song et al. (2005) derived a radius $=1.25 R_{\odot}$ based on the Hipparcos parallax of 10.88 mas, and temperature $6000 \mathrm{~K}$. Because of its low metallicity $([\mathrm{Fe} / \mathrm{H}]=-0.43, \S 2.3)$, the Hipparcos $B-V=0.56$ should be corrected to a solar metallicity $B-V$ index according to an expression given in Gray (1994); the result is $B-V=0.607$, corresponding to a temperature of 
$\sim 5900 \mathrm{~K}$. With this effective temperature, the monochromatic Hipparcos $V \operatorname{mag}(=8.98)$, and the Hipparcos parallax, we derive a single-star radius of $1.3 R_{\odot}$, in good agreement with the results of Song et al. Van Leeuwen (2007) revised the Hipparcos parallax to 10.4 mas, which corresponds to a single-star radius of $1.36 R_{\odot}$. With the simplifying assumption that the two stars in $\mathrm{BD}+20307$ are of equal magnitude, then their representative radii would each be $0.96 R_{\odot}$. (More precise values for the radii, appropriate for the relative magnitudes given in $\S 2.3$, can easily be derived.) Conversion of $T$ and $R$ to stellar mass depends on the uncertain stellar age (see below). If the mass of the primary is $1 M_{\odot}$, then the orbital inclination $i=31^{\circ}$.

Our orbital period for a circular orbit (Table 3) agrees well with the period of 3.448 days derived by Weinberger (2008) for an assumed circular orbit. However, her systemic radial velocity, $\gamma=-8.4 \mathrm{~km} \mathrm{~s}^{-1}$, does not agree within the respective errors with our $-12.43 \mathrm{~km} \mathrm{~s}^{-1}$. From Weinberger's discussion, the quoted error on her value for $\gamma$ would be about $0.6 \mathrm{~km} \mathrm{~s}^{-1}$. Then, the $>6 \sigma$ discrepancy in radial velocities must be attributed to either an underestimate of measurement error or a variable radial velocity or both. To investigate the possibility of a variable radial velocity, we divided our Table 3 data set into two halves, each 22 days long. The result was $\gamma=-12.4 \mathrm{~km} \mathrm{~s}^{-1}$ for each half, with estimated uncertainties of 0.1 and $0.2 \mathrm{~km} \mathrm{~s}^{-1}$ for the first and second half, respectively. Thus, if the systemic radial velocity of BD+20 307 is variable, then it changed by about $4 \mathrm{~km} \mathrm{~s}^{-1}$ over the 117 day interval between the 2007 October observations of Weinberger and the first half of our data set but then essentially did not change over the 22 days that separated the two halves of our data. While this pattern seems quite peculiar, it cannot be ruled out a priori. We note that, in 2004 September, Song et al. (2005) measured a systemic radial velocity of $-11 \pm 1 \mathrm{~km} \mathrm{~s}^{-1}$. Tokovinin et al. (2006) investigated spectroscopic binaries for the presence of tertiary companions. For a system with orbital period of 3.4 days, the probability is $\sim 70 \%$ that a third star will also be present. The period distribution is such that, depending on mass ratios, if a third star at BD+20 307 exists, then it might be detected either through adaptive optics imaging or monitoring of the systemic radial velocity.

Our observed orbital period of 3.42 days is in reasonable agreement with our (less precisely) determined photometric period of 3.53 days, as would be expected for a system with synchronized rotation and revolution. As noted by Weinberger (2008 and references therein), for solar mass stars and an orbital period of 3.4 days, this synchronization would occur in $\sim 100 \mathrm{Myr}$, which is likely to be substantially less than the age of BD+20 307 (see below). Quasi-sinusoidal light curves are typical of spot-induced variability in magnetically active stars (e.g., Henry et al. 1995; Henry \& Winn 2008). The low photometric amplitude implies that the difference in spot filling factor over the observable hemisphere during a full rotation period is less than $1 \%$. The moderately small inclination of the rotation (orbital) axis, $\sim 30^{\circ}$, would be consistent with the low photometric amplitude of the light curve. Midinfrared interferometric observations, for example with the Keck Nuller Interferometer, might confirm or disprove whether the debris disk around $\mathrm{BD}+20307$ lies in the orbital plane of the binary.

For main-sequence stars with outer convection zones, both rotation rate and associated stellar activity (e.g., X-ray flux, optical emission lines) can be used to derive stellar ages (see, for example, Gaidos 1998; Zuckerman \& Song 2004; Barnes 2007). While the rotation rate of $\mathrm{BD}+20307$ is controlled by its orbital period and not by its age, it is nonetheless worthwhile to characterize the binary's X-ray luminosity.
With the effective single-star radius and temperature calculated in the second paragraph of the present section and the Chandra-measured X-ray flux in the $0.5-2.0 \mathrm{keV}$ band quoted in $\S 2.1$, we calculate a fractional X-ray luminosity $\left(L_{\mathrm{X}} / L_{\mathrm{bol}}\right)$ of $1.6 \times 10^{-5}$. This may be compared to the upper limit to the ROSAT-measured X-ray flux over a similar energy band quoted by Song et al. (2005), i.e., $1.26 \times 10^{-5}$. Thus, it appears that the (presumably somewhat variable) X-ray flux from BD+20 307 was just below the ROSAT detection limit. The high X-ray flux and the rapid rotation period of 3.4 days are consistent with the fact that this system is a close, synchronized binary.

In the absence of rotation and activity age indicators, one can instead turn to lithium abundance, Galactic space motion $(U V W)$, metallicity, and placement on evolutionary tracks on an HR diagram. With the lithium absorption feature equivalent width (EW) given by Weinberger (2008) and our observed ratio of the two stellar continua $(=0.78)$ at $6708 \AA$, we derive an EW of $62 \mathrm{~m} \AA$ for the primary. This would be consistent with an age perhaps as young as stars in the UMa or Hyades groups (i.e., 500 Myr, e.g., Zuckerman et al. 2006), but also consistent with early G-type stars in the 2 Gyr old NGC 752, IC 4651, and NGC 3680 open clusters (Sestito et al. 2004). Indeed, this EW is even similar to that of early G-type stars in the 6-8 Gyr old NGC 188 (Randich et al. 2003). Then there is the secondary to consider, for which the $3 \sigma \mathrm{EW}$ is $<14 \mathrm{~m} \AA$ (Weinberger 2008). Such small EW have been measured for some early G-type stars as old as 5 Gyr, for example M67 (Sestito \& Randich (2005) and the Sun, and perhaps occasionally also as young as 2 Gyr (NGC 752; Sestito et al. 2004). Thus, one can conclude that the lithium lines in the two stars are probably most consistent with an age of a few Gyr or more.

With the van Leeuwen (2007) parallax, the Tycho 2 proper motion, and a radial velocity of $-12.4 \mathrm{~km} \mathrm{~s}^{-1}$, we calculate the Galactic space motion of $\mathrm{BD}+20307$ with respect to the Sun $\left(U V W=-3.1,-24.0,+4.8 \mathrm{~km} \mathrm{~s}^{-1}\right)$. This $U V W$ is more similar to the $U V W$ of moving groups with ages $\leq 100 \mathrm{Myr}$ than it is to the $U V W$ of older moving groups such as Ursa Majoris and the Hyades (see, e.g., Table 7 in Zuckerman \& Song 2004). While relatively few stars of age several Gyr or more have such small absolute values of all three space motion components, it is of course possible that $\mathrm{BD}+20307$ is one such star.

The low metallicity of BD+20 307 suggests an old age. Even the five open clusters with ages between 2 and 8 Gyr listed by Sestito \& Randich (2005) all have metallicities not much different than solar. ${ }^{7}$ With the evolutionary tracks presented by Nordstrom et al. (2004; their Fig. 12, [Fe/H $=-0.50]$ panel), and the van Leeuwen (2007) parallax, BD+20 307 looks to be many Gyr old. However, the parallax error is sufficiently large, $12 \%$, that within the uncertainties, a much younger age is consistent with these tracks. A very accurate measurement of the parallax of $\mathrm{BD}+20307$ would be worthwhile.

In summary, various methods taken together seem to imply a $\mathrm{BD}+20307$ age of least a few Gyr, and possibly considerably more. It is unlikely that the extraordinary quantity of warm dust at BD+20 307 has any direct relationship with the origin of terrestrial planets.

\footnotetext{
7 Lambda Boo stars have low abundances of refractory elements and dusty/ gaseous disks and are believed to be young, age less than or equal to a few $100 \mathrm{Myr}$. Their spectral types, late B to early F, are substantially earlier than that of $\mathrm{BD}+20307$. Thus, $\mathrm{BD}+20307$ is very unlikely to be a (young) $\lambda$ Boo-type star.
} 


\section{CONCLUSIONS}

Until new techniques are available to image or otherwise detect planets with masses and semimajor axes similar to those of the terrestrial planets of our solar system, we must rely on more indirect observations and models. One promising indirect approach is detection of dusty debris associated with terrestrial planet formation (e.g., Gorlova et al. 2007; Currie et al. 2007; Rhee et al. 2008; Lisse et al. 2008). To delineate the full extent of the era of terrestrial planet formation it is necessary to identify main-sequence stars with copious quantities of warm dust grains and ages of tens to many hundreds of millions of years. Thus, the goal of our research project was to establish the age of $\mathrm{BD}+20307$, which along with HD 23514 (an F6 member of the Pleiades), is one of the only two stars known with age $\geq 100 \mathrm{Myr}$ and strong indications of the existence of orbiting terrestrial planets (Rhee et al. 2008).

Based on the findings of our study, it now appears likely that BD+20 307 is an old binary star and its copious quantity of warm orbiting dust has no direct relationship with the era of planet formation. Rhee et al. (2008) present a moderately detailed discussion of the origin and fate of the dust at HD 23514 and $\mathrm{BD}+20307$. Given the large quantity of dust at BD+20 307, its short lifetime in orbit, and its concentration to a narrow range of warm temperatures (Weinberger et al. 2008), it is hard to escape the conclusion that something with the mass of a terrestrial planet was involved in a catastrophic collision, independent of whether that collision took place between two isolated objects or inside some sort of massive asteroid belt. A remarkable aspect of
$\mathrm{BD}+20307$ is that this collision apparently took place so late in its history. Perhaps, as suggested by the discussion of possible systemic radial velocity variations in $\S 3$, there is an unseen stellar or brown dwarf object present whose gravitational influence recently destabilized the orbits of rocky objects of planetary mass.

In any event, whatever its age, if the massive amounts of dust at $\mathrm{BD}+20307$ do point toward the presence of terrestrial planets, then this represents the first known example of planets of any mass in orbit around close binary stars, because the precision radial velocity technique is not sensitive to planets in such systems.

Because of its only modest sensitivity, IRAS was quite limited in the distance out to which it could detect warm dust at lowluminosity, main-sequence stars of K and M type. Should they exist, such very dusty, low-luminosity stars will be detectable by the Wide-Field Infrared Survey Explorer (WISE) mission. A substantial sample of very dusty stars of known age could delineate the era of terrestrial planet formation while perhaps revealing the existence of additional remarkable stars like BD+20 307.

We thank Inseok Song for a helpful conversation and Alycia Weinberger for calling the binary nature of BD+20 307 to our attention before publication. We thank the second referee for constructive comments. This research was supported in part by NASA through a Chandra Observatory award to UCLA. Automated Astronomy at Tennessee State University is supported by NASA, NSF, Tennessee State University, and the State of Tennessee through its Centers of Excellence program.
Arnaud, K. A. 1996, ASP Conf. Ser. 101, Astronomical Data Analysis Software and Systems V, ed. G. Jacoby \& J. Barnes (San Francisco: ASP), 17

Barker, E. S., Evans, D. S., \& Laing, J. D. 1967, R. Obs. Bull., 130

Barnes, S. A. 2007, ApJ, 669, 1167

Batten, A. H., Fletcher, J. M., \& MacCarthy, D. G. 1989, Publ. Dominion Astrophys. Obs., 17, 1

Bryden, G., et al. 2006, ApJ, 636, 1098

Cash, W. 1979, ApJ, 228, 939

Cox, A. N. 2000, Allen's Astrophysical Quantities (New York: Springer), 527

Currie, T., Kenyon, S. J., Rieke, G., Balog, Z., \& Bromley, B. C. 2007, ApJ, 663, L105

Eaton, J. A., Henry, G. W., \& Fekel, F. C. 2003, in The Future of Small Telescopes in the New Millennium, Volume II: The Telescopes We Use, ed. T. D. Oswalt (Dordrecht: Kluwer), 189

Eaton, J. A., \& Williamson, M. H. 2004, Proc. SPIE, 5496, 710

2007, PASP, 119,886

Flower, P. J. 1996, ApJ, 469, 355

Freeman, P. E., Kashyap, V., Rosner, R., \& Lamb, D. Q. 2002, ApJS, 138, 185

Gaidos, E. J. 1998, PASP, 110, 1259

Gorlova, N., et al. 2007, ApJ, 670, 516

Gray, D. 1994, PASP, 106, 1248

Henry, G. W. 1999, PASP, 111, 845

Henry, G. W., Fekel, F. C., \& Hall, D. S. 1995, AJ, 110, 2926

Henry, G. W., \& Winn, J. N. 2008, AJ, 135, 68

Hines, D., et al. 2006, ApJ, 638, 1070

Hong, J., van den Berg, M., Schlegel, E. M., Grindlay, J. E., Koenig, X., Laycock, S., \& Zhao, P. 2005, ApJ, 635, 907

Lallement, R., Welsh, B., Vergely, J., Crifo, F., \& Sfeir, D. 2003, A\&A, 411, 447

Lisse, C. M., Chen, C. H., Wyatt, M. C., \& Morlok, A. 2008, ApJ, 673, 1106 Lucy, L. B., \& Sweeney, M. A. 1971, AJ, 76, 544

Moore, C. E., Minnaert, M. G. J., \& Houtgast, J. 1966, NBS Monogr. 61, The Solar Spectrum $2935 \AA$ to $8770 \AA$ (Washington, D C: USGPO)
Nordstrom, B., et al. 2004, A\&A, 418, 989

Perryman, M. A. C., et al. 1997, The Hipparcos and Tycho Catalogues (ESA SP-1200; Noordwijk: ESA)

Petrie, R. M. 1939, Publ. Dominion Astrophys. Obs. Victoria, 7, 205

Radick, R. R., Thompson, D. T., Lockwood, G. W., Duncan, D. K., \& Baggett, W. E. 1987, ApJ, 321, 459

Randich, S., Sestito, P., \& Pallavicini, R. 2003, A\&A, 399, 133

Rhee, J. H., Song, I., \& Zuckerman, B. 2008, ApJ, 675, 777

Scarfe, C. D., Batten, A. H., \& Fletcher, J. M. 1990, Publ. Dominion Astrophys. Obs., 18, 21

Sestito, P., \& Randich, S. 2005, A\&A, 442, 615

Sestito, P., Randich, S., \& Pallavicini, R. 2004, A\&A, 426, 809

Smith, R., Wyatt, M. C., \& Dent, W. R. F. 2008, A\&A, 485, 897

Song, I., Zuckerman, B., Weinberger, A. J., \& Becklin, E. E. 2005, Nature, 436, 363

Su et al. 2006, ApJ, 653, 675

Taylor, B. J. 2003, A\&A, 398, 731

.2005, ApJS, 161, 444

Tokovinin, A., Thomas, S., Sterzik, M., \& Udry, S. 2006, A\&A, 450, 681

Trilling, D. E., et al. 2007, ApJ, 658, 1289

2008, ApJ, 674, 1086

Vaníček, P. 1971, Ap\&SS, 12, 10

van Leeuwen, F. 2007, A\&A, 474, 653

Weinberger, A. 2008, ApJ, 679, L41

Weinberger, A., et al. 2008, ApJ, submitted

Weisskopf, M. C., Brinkman, B., Canizares, C., Garmire, G., Murray, S., \& van Speybroeck, L. P. 2002, PASP, 114, 1

Welsh, B., Crifo, F., \& Lallement, R. 1998, A\&A, 333, 101

Wolfe, R. H., Horak, H. G., \& Storer, N. W. 1967, in Modern Astrophysics, ed. M. Hack (New York: Gordon \& Breach), 251

Zuckerman, B., Bessell, M. S., Song, I., \& Kim, S. 2006, ApJ, 649, L115

Zuckerman, B., \& Song, I. 2004, ARA\&A, 42, 685 